\title{
Note on holographic nonrelativistic Goldstone bosons
}

\author{
Riccardo Argurio, ${ }^{1}$ Andrea Marzolla, ${ }^{1}$ Andrea Mezzalira, ${ }^{2,3}$ and Daniel Naegels ${ }^{1}$ \\ ${ }^{1}$ Physique Théorique et Mathématique and International Solvay Institutes, Université Libre de Bruxelles, \\ C.P. 231, 1050 Brussels, Belgium \\ ${ }^{2}$ Institute Lorentz for Theoretical Physics, Leiden University, P.O. Box 9506, \\ Leiden 2300RA, The Netherlands \\ ${ }^{3}$ School of Mathematics, Trinity College, Dublin 2, Ireland
}

(Received 8 July 2015; published 24 September 2015)

\begin{abstract}
We consider a holographic setup where relativistic invariance is broken by a chemical potential, and a non-Abelian internal symmetry is broken spontaneously. We use the tool of holographic renormalization in order to infer what can be learned purely by analytic boundary considerations. We find that the expected Ward identities are correctly reproduced. In particular, we obtain the identity which implies the noncommutation of a pair of broken charges, which leads to the presence of Goldstone bosons with quadratic dispersion relations.
\end{abstract}

DOI: 10.1103/PhysRevD.92.066009

PACS numbers: 11.25.Tq, 14.80.Va, 11.10.Gh

\section{INTRODUCTION}

Goldstone's theorem has been originally proved in a Lorentz-invariant formulation. Its extension to nonrelativistic settings is not straightforward and raises a problem of counting, as was quite soon realized [1]: the number of massless modes does not match the number of broken generators in this context, and moreover the dispersion relation of nonrelativistic Goldstone bosons is not any more constrained to be linear with constant of proportionality fixed to the speed of light, as in the Lorentz-invariant case. Nonrelativistic setups occur quite often in condensed matter physics, but the issue of the generalization of Goldstone's theorem to these setups has obviously great theoretical interest and only recently some main developments have been achieved in its understanding [2-5]. The occurrence of Goldstone bosons with quadratic dispersion relation has been related to the presence of pairs of broken generators whose commutator has a nontrivial vacuum expectation value (VEV), which is forbidden in the case of Lorentz invariance. Such Goldstone bosons with quadratic dispersion relations are usually accompanied by a massive partner whose mass is proportional to the amount of Lorentz breaking. Eventually, there can still be massless particles which have linear dispersion relations, as in the relativistic case, but with a model dependent velocity.

The aim of this note is to explore in a holographic setup [6-8] what can be learned on these different kinds of light modes. In particular, we focus on what can be extracted $a$ priori in a given model, i.e. just by specifying how the symmetries are broken. The holographic model is supposed to represent a theory with a large number of degrees of freedom and at strong coupling, which displays a pattern of symmetry breaking allowing for the various types of Goldstone bosons to be present. We study a model with the minimal requirements in order to expect all these light modes, namely a $U(2)$ global symmetry which will be spontaneously broken, with a source for the timelike component of the Abelian $U(1)$ current, i.e. a chemical potential, which explicitly breaks boost invariance [but preserves $U(2)$ ]. This model has been previously considered in [9], where the system was set at finite temperature and numerical techniques were essentially employed to analyze it (see also [10] for a different holographic model). We would rather consider the model at zero temperature, in analogy with the original field theory model presented in [11,12], and perform a purely boundary analysis through analytical techniques.

For this aim we will employ the technique of holographic renormalization $[13,14]$ in order to deduce the presence of such Goldstone bosons purely by symmetry arguments. In practical terms, the Ward identities for broken symmetries [15] establish the existence of massless modes generated by the broken currents. Moreover in the nonrelativistic case, when commutators of broken generators have VEVs, it can be shown that some of these massless modes must have quadratic dispersion relations [16]. By carefully performing the procedure of obtaining the renormalized action of the holographic model, we show that such Ward identities are exactly reproduced. Unfortunately, this is all we can extract from a purely boundary analysis. This allows us to determine the existence of different types of Goldstone bosons and to establish if their dispersion relation is either linear $\left(\omega=c_{s} k\right.$, with $c_{s}$ "sound" velocity) or quadratic $\left(\omega=\frac{k^{2}}{M}\right.$, with $M$ being a parameter with the dimensions of a mass), but the exact values of the constant factors $c_{s}$ and $M$ remain undetermined. In order to explicitly derive the dispersion relations and quantitatively determine these factors, an analysis of bulk fluctuations is required, 
similarly as it is necessary in order to determine the gap towards the massive excitations.

The article is structured as follows. We first present the holographic model and derive the renormalized action. Then by paying attention to gauge invariance, we extract the relevant correlators and find that they satisfy the expected Ward identities. We end by commenting on how to go beyond. In Appendix A we consider an alternative gauge fixing of the holographic model; in Appendix B we show in the field theory model of $[11,12]$ how the nonlocal parts of the correlators, which can be explicitly computed, conspire to satisfy the same Ward identities, while yielding the expected dispersion relations.

\section{A HOLOGRAPHIC MODEL FOR NONRELATIVISTIC SYMMETRY BREAKING}

We now outline the model discussed in [9]. This is a typically strongly coupled theory represented by its holographic dual. We assume that the theory has a nontrivial UV conformal fixed point. In this UV CFT, we focus on the conserved currents $J_{i}^{a}$ which form a $U(2)$ algebra, and on a relevant operator $\mathcal{O}_{\Psi}$ which is a doublet of $U(2)$. In the holographic dual, this means that we need to have a bulk theory in an asymptotically anti-de Sitter (AdS) spacetime, which includes dynamical $U(2)$ gauge fields and a complex doublet scalar with negative squared mass. Since we are not interested in computations involving the stress energy tensor, we set gravity in the bulk not to be dynamical. We thus consider the following bulk action, where for simplicity we choose four bulk dimensions (and hence three dimensions for the boundary theory $)^{1}$ :

$$
\begin{aligned}
S_{\text {bulk }}= & \int d^{4} x \sqrt{-g} \\
& \times\left\{-\frac{1}{4} F_{\mu \nu}^{a} F^{a \mu \nu}-D_{\mu} \Psi^{\dagger} D^{\mu} \Psi-m^{2} \Psi^{\dagger} \Psi\right\},
\end{aligned}
$$

where $F_{\mu \nu}^{a}=\partial_{\mu} A_{\nu}^{a}-\partial_{\nu} A_{\mu}^{a}+f^{a b c} A_{\mu}^{b} A_{\nu}^{c}$ and $D_{\mu} \Psi=\partial_{\mu} \Psi-$ $i A_{\mu}^{a} T^{a} \Psi$. The field $\Psi \equiv\left(\begin{array}{c}\lambda \\ \phi\end{array}\right)$ is a complex scalar doublet, while $T^{a}$, with $a=\{0,1,2,3\}$, are the generators of $U(2)$ in the fundamental representation, namely $T^{0}=\frac{1}{2} \mathbb{1}$ and $T^{a}=\frac{1}{2} \tau^{a}$ otherwise, with $\tau^{a}$ being the Pauli matrices, and they satisfy $\left[T^{a}, T^{b}\right]=i f^{a b c} T^{c}$, with $f^{a b c}=0$ if any of the indices is 0 or $f^{a b c} \equiv \epsilon^{a b c}$ otherwise.

The metric is fixed to be the AdS one, defined by

$$
d s^{2}=\frac{1}{z^{2}}\left(\eta_{i j} d x_{i} d x_{j}+d z^{2}\right)
$$

\footnotetext{
${ }^{1}$ We argue later that the choice of higher dimensions should not qualitatively modify our results.
}

where $\eta_{i j}$ is the mostly plus Minkowski metric. Note that since this is pure AdS, on the field theory side it corresponds to considering zero temperature, in contrast with the analysis of [9] where a nonzero temperature was always required.

We will not consider any backreaction on the metric. This is motivated by the fact that we in any case restrict our attention to the near-boundary region, where backreaction effects can be shown to be subleading and actually completely irrelevant to our considerations.

Let us first consider the field configuration defining the background, and thus the vacuum of the dual theory. First of all, as anticipated, the theory is nonrelativistic because boost invariance is broken by the presence of a chemical potential, $\mathcal{L}_{\mathrm{QFT}} \supset \mu J_{t}^{0}$. This means that there should be a nontrivial source for $J_{t}^{0}$, namely $A_{t}^{0}$ should have a leading mode turned on. This source breaks Lorentz invariance, but preserves all of $U(2)$ since the $U(1)$ generator commutes with all the algebra. In the vacuum of the theory, we expect that nontrivial dynamics generates VEVs for the operator $\mathcal{O}_{\Psi}$, thus breaking $U(2)$ to $U(1)$. For simplicity we take this $U(1)$ to be the one generated by $T^{0}+T^{3}$. The unbroken symmetries allow for VEVs to be generated also for $J_{t}^{0}$ and $J_{t}^{3}$. The latter is crucial for obtaining VEVs for commutators of charges, and thus for the appearance of Goldstone bosons with quadratic dispersion relations. In the bulk, we thus have subleading modes for the profiles of $A_{t}^{0}$, $A_{t}^{3}$ and for $\Psi$ in its bottom component.

From now on, we fix the dimension of $\mathcal{O}_{\Psi}$ to be two, which implies $m^{2}=-2$ (in units of the AdS radius). The background profiles are thus the following:

$\left.\phi\right|_{B}=\bar{\phi} z^{2},\left.\quad A_{t}^{0}\right|_{B}=\mu+\bar{A}^{0} z,\left.\quad A_{t}^{3}\right|_{B}=\bar{A}^{3} z$,

where we take $\bar{\phi}, \mu, \bar{A}^{0}$ and $\bar{A}^{3}$ to be real constants, and all the other fields vanish. These profiles satisfy the free equations of motion. As with the metric, the backreaction of the above profiles on each other can be safely neglected. ${ }^{2}$ Strictly speaking, without backreaction the leading and subleading modes can be chosen independently. In particular $\bar{A}^{0,3}$ do not depend on $\mu$, which could be even set to zero. However this would mean that some physical mechanism should generate spontaneously a Lorentz breaking VEV such as $\left\langle J_{t}^{3}\right\rangle$. In the following we rather assume that $\mu \neq 0$ even if it is not technically necessary.

We now proceed to fluctuate the fields over the background. The aim is to obtain the on-shell action up to quadratic order, since we are interested in

\footnotetext{
${ }^{2}$ The backreaction would intervene at $\mathcal{O}\left(z^{4}\right)$ in $\phi$ and at $\mathcal{O}\left(z^{2}\right)$ in $A_{t}^{0,3}$, hence at orders that do not contribute to the rest of our computations.
} 
two- and one-point functions. At this order, the on-shell action reduces to a boundary term. In the following, we fix as usual the axial gauge $A_{z}^{a}=0$. The linearized equations of motion are the following (we use the notation $i=\{t, x, y\}$ and $u=\{x, y\}$, and we label the fluctuations above the background in the same way as the field itself). The constraint coming from the variation with respect to $A_{z}^{a}$ reads

$$
\begin{aligned}
& z \partial_{z} \partial_{i} A_{i}^{a}-z \bar{A}^{3} f^{3 a b}\left(A_{t}^{b}-z \partial_{z} A_{t}^{b}\right)-i \bar{\phi}\left(T^{a}\right)_{21}\left(2 \lambda-z \partial_{z} \lambda\right) \\
& \quad+i \bar{\phi}\left(T^{a}\right)_{12}\left(2 \lambda^{\dagger}-z \partial_{z} \lambda^{\dagger}\right)-i \bar{\phi}\left(T^{a}\right)_{22}\left(2\left(\phi-\phi^{\dagger}\right)\right. \\
& \left.\quad-z \partial_{z}\left(\phi-\phi^{\dagger}\right)\right)=0 .
\end{aligned}
$$

The equations of motion for the vector field fluctuations, separated in temporal and spatial components, are

$$
\begin{aligned}
& z^{2} \partial_{z}^{2} A_{t}^{a}+z^{2} \partial_{u}^{2} A_{t}^{a}-z^{2} \partial_{t} \partial_{u} A_{u}^{a}+z^{3} f^{a b 3} \bar{A}^{3} \partial_{u} A_{u}^{b} \\
& \quad+i z^{2} \bar{\phi}\left[\left(T^{a}\right)_{12} \partial_{t} \lambda^{\dagger}-\left(T^{a}\right)_{21} \partial_{t} \lambda-\left(T^{a}\right)_{22} \partial_{t}\left(\phi-\phi^{\dagger}\right)\right] \\
& \quad-z^{2}\left(\mu+\bar{A}^{0} z\right) \bar{\phi}\left[\left(T^{a}\right)_{21} \lambda+\left(T^{a}\right)_{12} \lambda^{\dagger}+\left(T^{a}\right)_{22}\left(\phi+\phi^{\dagger}\right)\right] \\
& \quad-z^{3} \bar{A}^{3} \bar{\phi}\left[\left\{T^{a}, T^{3}\right\}_{21} \lambda+\left\{T^{a}, T^{3}\right\}_{12} \lambda^{\dagger}\right. \\
& \left.\quad+\left\{T^{a}, T^{3}\right\}_{22}\left(\phi+\phi^{\dagger}\right)\right]-z^{4} \bar{\phi}^{2} A_{t}^{b}\left\{T^{a}, T^{b}\right\}_{22}=0,
\end{aligned}
$$

$$
\begin{aligned}
& z^{2} \partial_{z}^{2} A_{u}^{a}+z^{2} \partial_{i}^{2} A_{u}^{a}-z^{2} \partial_{u} \partial_{i} A_{i}^{a}+2 z^{3} f^{a b 3} \bar{A}^{3} \partial_{t} A_{u}^{b} \\
& \quad-z^{3} f^{a b 3} \bar{A}^{3} \partial_{u} A_{t}^{b}-z^{4}\left(\bar{A}^{3}\right)^{2} f^{a b 3} f^{b c 3} A_{u}^{c} \\
& \quad+i z^{2} \bar{\phi}\left[\left(T^{a}\right)_{12} \partial_{u} \lambda^{\dagger}-\left(T^{a}\right)_{21} \partial_{u} \lambda-\left(T^{a}\right)_{22} \partial_{u}\left(\phi-\phi^{\dagger}\right)\right] \\
& \quad-z^{4} \bar{\phi}^{2} A_{u}^{b}\left\{T^{a}, T^{b}\right\}_{22}=0 .
\end{aligned}
$$

Finally, the equations for the scalar fluctuations are

$$
\begin{aligned}
& z^{2} \partial_{z}^{2} \lambda-2 z \partial_{z} \lambda+z^{2} \partial_{i}^{2} \lambda+2 \lambda-i z^{4} \bar{\phi}\left(T^{a}\right)_{12} \partial_{i} A_{i}^{a} \\
& \quad+i z^{2}\left(\mu+\bar{A}^{0} z+\bar{A}^{3} z\right) \partial_{t} \lambda+\frac{1}{4} z^{2}\left(\mu+\bar{A}^{0} z+\bar{A}^{3} z\right)^{2} \lambda \\
& \quad+z^{4}\left(\mu+\bar{A}^{0} z\right) \bar{\phi}\left(T^{a}\right)_{12} A_{t}^{a}=0, \\
& z^{2} \partial_{z}^{2} \phi-2 z \partial_{z} \phi+z^{2} \partial_{i}^{2} \phi+2 \phi-i z^{4} \bar{\phi}\left(T^{a}\right)_{22} \partial_{i} A_{i}^{a} \\
& \quad+i z^{2}\left(\mu+\bar{A}^{0} z-\bar{A}^{3} z\right) \partial_{t} \phi+\frac{1}{4} z^{2}\left(\mu+\bar{A}^{0} z-\bar{A}^{3} z\right)^{2} \phi \\
& \quad+z^{4}\left(\mu+\bar{A}^{0} z\right) \bar{\phi}\left(T^{a}\right)_{22} A_{t}^{a}+z^{5} \bar{A}^{3} \bar{\phi}\left\{T^{a}, T^{3}\right\}_{22} A_{t}^{a}=0 .
\end{aligned}
$$

The last two equations should be supplemented by their complex conjugates.

The on-shell action is obtained expanding (2.1) up to quadratic order in the fluctuations, and then substituting the equations of motion, possibly integrating by parts.
At the regularizing surface $z=\epsilon$, we obtain

$$
\begin{aligned}
S_{\mathrm{reg}}= & -\int_{z=\epsilon} d^{3} x\left\{\bar{A}^{0} A_{t}^{0}+\bar{A}^{3} A_{t}^{3}-\frac{2}{z} \bar{\phi}\left(\phi+\phi^{\dagger}\right)\right. \\
& +\frac{1}{2} A_{t}^{a} \partial_{z} A_{t}^{a}-\frac{1}{2} A_{u}^{a} \partial_{z} A_{u}^{a} \\
& \left.-\frac{1}{2 z^{2}}\left(\lambda^{\dagger} \partial_{z} \lambda+\lambda \partial_{z} \lambda^{\dagger}+\phi^{\dagger} \partial_{z} \phi+\phi \partial_{z} \phi^{\dagger}\right)\right\} .
\end{aligned}
$$

At this point, we note that the quadratic terms are exactly the same ones that would arise in a configuration with vanishing backgrounds. The presence of nontrivial backgrounds must then show up when expanding the fluctuations near the boundary as powers of $z$. There is however one more substitution that we can make, which makes the dependence on the background manifest even before expanding the fluctuations. We can indeed also substitute Eq. (2.4) which only has first order derivatives in $z$. However in order to perform the substitution we also have to split the vector into its irreducible components. We choose here to split into transverse and longitudinal parts with respect to the spatial coordinates $u$. Thus we keep $A_{t}^{a}$ as the temporal component, while we split $^{3}$

$$
A_{u}^{a}=A_{u}^{T a}+\partial_{u} A^{L a}, \quad \partial_{u} A_{u}^{T a}=0 .
$$

We eventually arrive at

$$
\begin{aligned}
S_{\text {reg }}= & -\int_{z=e} d^{3} x\left\{\bar{A}^{0} A_{t}^{0}+\bar{A}^{3} A_{t}^{3}-\frac{2}{z} \bar{\phi}\left(\phi+\phi^{\dagger}\right)\right. \\
& +\frac{1}{2} A_{t}^{a} \partial_{z} A_{t}^{a}-\frac{1}{2} A_{u}^{T a} \partial_{z} A_{u}^{T a} \\
& +\frac{1}{2} A^{L a} \partial_{z} \partial_{t} A_{t}^{a}+\frac{1}{2} \bar{A}^{3} f^{a b 3} A^{L a}\left(A_{t}^{b}-z \partial_{z} A_{t}^{b}\right) \\
& +\frac{i}{2 z} \bar{\phi} A^{L a}\left[\left(T^{a}\right)_{21}\left(2 \lambda-z \partial_{z} \lambda\right)-\left(T^{a}\right)_{12}\left(2 \lambda^{\dagger}-z \partial_{z} \lambda^{\dagger}\right)\right. \\
& \left.+\left(T^{a}\right)_{22}\left(2 \phi-2 \phi^{\dagger}-z \partial_{z} \phi+z \partial_{z} \phi^{\dagger}\right)\right] \\
& \left.-\frac{1}{2 z^{2}}\left(\lambda^{\dagger} \partial_{z} \lambda+\lambda \partial_{z} \lambda^{\dagger}+\phi^{\dagger} \partial_{z} \phi+\phi \partial_{z} \phi^{\dagger}\right)\right\} . \quad(2.11)
\end{aligned}
$$

We now consider the near-boundary expansion of the fluctuating fields:

\footnotetext{
${ }^{3}$ In Appendix A we discuss a different splitting, where the transverse and longitudinal parts are taken with respect to the spacetime coordinates $i$.
} 


$$
\begin{aligned}
A_{t}^{a} & =A_{t(0)}^{a}+A_{t(1)}^{a} z+\cdots \\
A_{u}^{T a} & =A_{u(0)}^{T a}+A_{u(1)}^{T a} z+\cdots \\
A^{L a} & =A_{(0)}^{L a}+A_{(1)}^{L a} z+\cdots \\
\lambda & =\lambda_{(0)} z+\lambda_{(1)} z^{2}+\cdots \\
\phi & =\phi_{(0)} z+\phi_{(1)} z^{2}+\cdots
\end{aligned}
$$

Note that all the modes with (0) subscript are the ones which are usually considered as sources, while all the ones with (1) subscript coincide with the independent subleading modes, to be identified with the VEVs of the fluctuations. The latter occur at the next-to-leading order in the $z$ expansion because of the fact that we are considering conserved currents and an operator of dimension 2 in a three-dimensional boundary theory.

The divergent terms that one finds in $S_{\text {reg }}$ are taken care of by adding counterterms, which are independent of the presence of the profile. Again, due to the involved dimensions, no finite counterterms arise and hence no scheme dependence. ${ }^{4}$ Eventually, the renormalized action is the following:

$$
\begin{aligned}
S_{\text {ren }}= & -\int d^{3} x\left\{\bar{A}^{0} A_{t(0)}^{0}+\bar{A}^{3} A_{t(0)}^{3}-2 \bar{\phi}\left(\phi_{(0)}+\phi_{(0)}^{\dagger}\right)\right. \\
& +\frac{1}{2} A_{t(0)}^{a} A_{t(1)}^{a}-\frac{1}{2} A_{u(0)}^{T a} A_{u(1)}^{T a}-\frac{1}{2} \partial_{t} A_{(0)}^{L a} A_{t(1)}^{a} \\
& +\frac{1}{2} \bar{A}^{3} f^{a b 3} A_{(0)}^{L a} A_{t(0)}^{b}+\frac{i}{2} \bar{\phi} A_{(0)}^{L a}\left[\left(T^{a}\right)_{21} \lambda_{(0)}-\left(T^{a}\right)_{12} \lambda_{(0)}^{\dagger}\right. \\
& \left.+\left(T^{a}\right)_{22}\left(\phi_{(0)}-\phi_{(0)}^{\dagger}\right)\right]-\frac{1}{2}\left(\lambda_{(0)}^{\dagger} \lambda_{(1)}+\lambda_{(0)} \lambda_{(1)}^{\dagger}\right. \\
& \left.\left.+\phi_{(0)}^{\dagger} \phi_{(1)}+\phi_{(0)} \phi_{(1)}^{\dagger}\right)\right\} .
\end{aligned}
$$

The first three terms of the above functional, linear in the fluctuations, just give us the VEVs of the associated operators $J_{t}^{0}, J_{t}^{3}$ and $\operatorname{Re} \mathcal{O}_{\phi}$. The other terms, quadratic in the fluctuations, contain in principle all the information about two-point functions. However, this information is encoded in the way the subleading modes with (1) subscript depend on the sources with ${ }_{(0)}$ subscript. This dependence is fixed through the bulk boundary conditions (generically, asking regularity) and is typically nonlocal, since it requires solving the equations for the fluctuations inside the bulk.

\footnotetext{
${ }^{4}$ If we had considered an operator of dimension 3 in a fourdimensional boundary theory, for instance, we would have logarithmical terms in the expansions of the fluctuations which would lead to a logarithmical divergence in the regularized action. The renormalization of the logarithmical terms carries along finite counterterms that would be scheme dependent. However, as it should be clear in the following, this ambiguity would not affect the terms we are interested in.
}

We now turn to see what can be extracted from $S_{\text {ren }}$ without solving the bulk equations of motion.

\section{WARD IDENTITIES FROM THE RENORMALIZED ACTION}

A brief inspection of $S_{\text {ren }}$ in (2.13) shows that there are two kinds of quadratic terms: those which are bilinears of a source and a VEV of the fluctuations and those which involve only sources. All the latter ones are proportional to a VEV of the nontrivial background we have chosen; thus they would not be present in a model with trivial profiles and only the former ones would survive. Nonetheless it turns out that terms of the second kind are also hidden in terms of the first kind, because of gauge invariance.

Consider indeed the bulk gauge invariance of the action (2.1), for which $\delta \Psi=i \alpha \Psi$ and $\delta A_{\mu}=\partial_{\mu} \alpha+$ $i\left[\alpha, A_{\mu}\right]$. Because of the gauge fixing $A_{z}^{a}=0$, the gauge parameter does not depend on $z$. It is then easy to see how the residual gauge transformations act on each mode in the expansions (2.12). Recalling further that we are only interested in the quadratic part of the action, we neglect terms in the gauge variations which are bilinear in the gauge parameter and the mode of the fluctuation. We are then left with

$$
\begin{aligned}
\delta \lambda_{(0)} & =0, & \delta \lambda_{(1)}=i \alpha^{a}\left(T^{a}\right)_{12} \bar{\phi}, \\
\delta \phi_{(0)} & =0, & \delta \phi_{(1)}=i \alpha^{a}\left(T^{a}\right)_{22} \bar{\phi}, \\
\delta A_{t(0)}^{a} & =\partial_{t} \alpha^{a}, & \delta A_{t(1)}^{a}=-f^{a b 3} \alpha^{b} \bar{A}^{3}, \\
\delta A_{u(0)}^{T a} & =0, & \delta A_{u(1)}^{T a}=0, \\
\delta A_{(0)}^{L a} & =\alpha^{a}, & \delta A_{(1)}^{L a}=0 .
\end{aligned}
$$

After solving the bulk equations of motion for the fluctuations, the subleading modes $\Phi_{(1)}$ will be expressed in terms of nonlocal functions of the sources $\Phi_{(0)}$. However, in order to solve the equations, we would have to impose boundary conditions in the bulk and, to preserve the gauge symmetry, we should take care of imposing them on gauge-invariant combinations of the fields. So we have to consider only gauge-invariant combinations of both the sources and the subleading modes, as for instance

$$
\begin{aligned}
& \lambda_{(1)}-i \bar{\phi}\left(T^{a}\right)_{12} A_{(0)}^{L a}, \\
& \phi_{(1)}-i \bar{\phi}\left(T^{a}\right)_{22} A_{(0)}^{L a}, \\
& A_{t(1)}^{a}+\bar{A}^{3} f^{a b 3} A_{(0)}^{L b}
\end{aligned}
$$

for the VEVs and

$$
A_{t(0)}^{a}-\partial_{t} A_{(0)}^{L a}
$$

for the sources. 
We are now allowed to assume the general relations between VEVs and sources:

$$
\begin{aligned}
\lambda_{(1)}= & i \bar{\phi}\left(T^{a}\right)_{12} A_{(0)}^{L a}+f(\partial) \lambda_{(0)}+\tilde{f}^{a}(\partial)\left(A_{t(0)}^{a}-\partial_{t} A_{(0)}^{L a}\right), \\
\phi_{(1)}= & i \bar{\phi}\left(T^{a}\right)_{22} A_{(0)}^{L a}+g(\partial) \phi_{(0)}+\tilde{g}^{a}(\partial)\left(A_{t(0)}^{a}-\partial_{t} A_{(0)}^{L a}\right), \\
A_{t(1)}^{a}= & -\bar{A}^{3} f^{a b 3} A_{(0)}^{L b}+h^{a b}(\partial)\left(A_{t(0)}^{b}-\partial_{t} A_{(0)}^{L b}\right) \\
& +k^{a}(\partial) \lambda_{(0)}+l^{a}(\partial) \phi_{(0)}+k^{a}(\partial)^{*} \lambda_{(0)}^{\dagger}+l^{a}(\partial)^{*} \phi_{(0)}^{\dagger}, \\
A_{u(1)}^{T a}= & m^{a b}(\partial) A_{u(0)}^{T b},
\end{aligned}
$$

where all the functions of $\partial$ collectively indicate expressions that are typically nonlocal in space and/or time derivatives, and that cannot be determined without solving the equations in the bulk. However, we see that in some combinations of the correlators the dependence on these unknown functions drops out.

We can now rewrite $S_{\text {ren }}$ eliminating all the VEVs, using the expressions above:

$$
\begin{aligned}
S_{\text {ren }}= & -\int d^{3} x\left\{\bar{A}^{0} A_{t(0)}^{0}+\bar{A}^{3} A_{t(0)}^{3}-2 \bar{\phi}\left(\phi_{(0)}+\phi_{(0)}^{\dagger}\right)-\frac{1}{2} A_{u(0)}^{T a} m^{a b}(\partial) A_{u(0)}^{T b}\right. \\
& +\frac{1}{2}\left(A_{t(0)}^{a}-\partial_{t} A_{(0)}^{L a}\right) h^{a b}(\partial)\left(A_{t(0)}^{b}-\partial_{t} A_{(0)}^{L b}\right)-\frac{1}{2} \bar{A}^{3} f^{a b 3}\left(2 A_{t(0)}^{a}-\partial_{t} A_{(0)}^{L a}\right) A_{(0)}^{L b} \\
& +i \bar{\phi} A_{(0)}^{L a}\left[\left(T^{a}\right)_{21} \lambda_{(0)}-\left(T^{a}\right)_{12} \lambda_{(0)}^{\dagger}+\left(T^{a}\right)_{22}\left(\phi_{(0)}-\phi_{(0)}^{\dagger}\right)\right] \\
& -\frac{1}{2} \lambda_{(0)}^{\dagger}\left(f(\partial)+f(\partial)^{*}\right) \lambda_{(0)}-\frac{1}{2} \phi_{(0)}^{\dagger}\left(g(\partial)+g(\partial)^{*}\right) \phi_{(0)} \\
& -\frac{1}{2}\left[\lambda_{(0)}^{\dagger}\left(\tilde{f}^{a}(\partial)+k^{a}(\partial)^{*}\right)+\lambda_{(0)}\left(\tilde{f}^{a}(\partial)^{*}+k^{a}(\partial)\right)\right]\left(A_{t(0)}^{a}-\partial_{t} A_{(0)}^{L a}\right) \\
& -\frac{1}{2}\left[\phi_{(0)}^{\dagger}\left(\left(\tilde{g}^{a}(\partial)+l^{a}(\partial)^{*}\right)+\phi_{(0)}\left(\tilde{g}^{a}(\partial)^{*}+l^{a}(\partial)\right)\right]\left(A_{t(0)}^{a}-\partial_{t} A_{(0)}^{L a}\right)\right\} .
\end{aligned}
$$

This is the generating functional for the one- and two-point functions in our theory. ${ }^{5}$ The precise relations between sources of operators in the boundary theory and modes of bulk fluctuations are the following. For the scalar operators we have

$$
\int_{\partial \mathrm{AdS}} d^{3} x\left(\lambda_{(0)} \mathcal{O}_{\lambda}+\phi_{(0)} \mathcal{O}_{\phi}+\text { c.c. }\right),
$$

while for the currents

$$
\int_{\partial \mathrm{AdS}} d^{3} x\left(A_{t(0)}^{a} J_{t}^{a}-A_{u(0)}^{T a} J_{u}^{T a}+A_{(0)}^{L a} \partial_{u} J_{u}^{a}\right),
$$

so that $A_{u(0)}^{T a}$ sources the purely transverse part of $J_{u}^{a}$ while $A_{(0)}^{L a}$ sources its longitudinal piece.

Some two-point functions will be entirely determined by their nonlocal part, for instance those with two transverse currents or two scalar operators, and we will have nothing to say about them since we do not solve the bulk equations. On the other hand, we see from the final expression of our generating functional $S_{\text {ren }}$ that some other two-point functions might be directly determined by our analysis. It should be the case for two-point functions involving the

\footnotetext{
${ }^{5}$ Note that the scheme ambiguity which would arise in higher dimensions would be contained in the possibility to redefine the nonlocal functions in the above expression.
}

temporal and longitudinal components of the currents, both among themselves or mixed with scalar operators. Indeed, local constant terms involving the sources of these operators appear in (3.5).

Let us list here a number of such correlators:

$$
\begin{aligned}
\left\langle J_{t}^{a}(x) J_{t}^{b}(y)\right\rangle & =-i \frac{\delta^{2} S_{\text {ren }}}{\delta A_{t(0)}^{a}(x) \delta A_{t(0)}^{b}}(y) \\
& =i h^{a b}(\partial) \delta^{3}(x-y),
\end{aligned}
$$

$$
\begin{aligned}
\left\langle J_{t}^{a}(x) \partial_{u} J_{u}^{b}(y)\right\rangle & =-i \frac{\delta^{2} S_{\text {ren }}}{\delta A_{t(0)}^{a}(x) \delta A_{(0)}^{L b}(y)} \\
& =-i\left[h^{a b}(\partial) \partial_{t}+\bar{A}^{3} f^{a b 3}\right] \delta^{3}(x-y),
\end{aligned}
$$

$$
\begin{aligned}
\left\langle\partial_{u} J_{u}^{a}(x) \partial_{v} J_{v}^{b}(y)\right\rangle & =-i \frac{\delta^{2} S_{\text {ren }}}{\delta A_{(0)}^{L a}(x) \delta A_{(0)}^{L b}(y)} \\
& =-i\left[h^{a b}(\partial) \partial_{t}^{2}+\bar{A}^{3} f^{a b 3} \partial_{t}\right] \delta^{3}(x-y),
\end{aligned}
$$

$$
\begin{aligned}
\left\langle J_{t}^{a}(x) \mathcal{O}_{\lambda}(y)\right\rangle & =-i \frac{\delta^{2} S_{\text {ren }}}{\delta A_{t(0)}^{a}(x) \delta \lambda_{(0)}(y)} \\
& =-\frac{i}{2}\left(\tilde{f}^{a}(\partial)^{*}+k^{a}(\partial)\right) \delta^{3}(x-y),
\end{aligned}
$$




$$
\begin{aligned}
\left\langle\partial_{u} J_{u}^{a}(x) \mathcal{O}_{\lambda}(y)\right\rangle= & -i \frac{\delta^{2} S_{\text {ren }}}{\delta A_{(0)}^{L a}(x) \delta \lambda_{(0)}(y)} \\
= & i\left[-\frac{1}{2}\left(\tilde{f}^{a}(\partial)^{*}+k^{a}(\partial)\right) \partial_{t}+i \bar{\phi}\left(T^{a}\right)_{21}\right] \\
& \times \delta^{3}(x-y), \\
\left\langle J_{t}^{a}(x) \mathcal{O}_{\phi}(y)\right\rangle=- & i \frac{\delta^{2} S_{\text {ren }}}{\delta A_{t(0)}^{a}(x) \delta \phi_{(0)}(y)} \\
= & -\frac{i}{2}\left(\tilde{g}^{a}(\partial)^{*}+l^{a}(\partial)\right) \delta^{3}(x-y), \\
\left\langle\partial_{u} J_{u}^{a}(x) \mathcal{O}_{\phi}(y)\right\rangle= & -i \frac{\delta^{2} S_{\text {ren }}}{\delta A_{(0)}^{L a}(x) \delta \phi_{(0)}(y)} \\
= & i\left[-\frac{1}{2}\left(\tilde{g}^{a}(\partial)^{*}+l^{a}(\partial)\right) \partial_{t}+i \bar{\phi}\left(T^{a}\right)_{22}\right] \\
& \times \delta^{3}(x-y) .
\end{aligned}
$$

We thus immediately see that some combinations are given entirely by the constant terms, or trivially vanish:

$$
-\left\langle\partial_{t} J_{t}^{a}(x) J_{t}^{b}(y)\right\rangle+\left\langle\partial_{u} J_{u}^{a}(x) J_{t}^{b}(y)\right\rangle=-i \bar{A}^{3} f^{a b 3} \delta^{3}(x-y),
$$

$$
\begin{gathered}
-\left\langle\partial_{t} J_{t}^{a}(x) \partial_{v} J_{v}^{b}(y)\right\rangle+\left\langle\partial_{u} J_{u}^{a}(x) \partial_{v} J_{v}^{b}(y)\right\rangle=0, \\
-\left\langle\partial_{t} J_{t}^{a}(x) \mathcal{O}_{\lambda}(y)\right\rangle+\left\langle\partial_{u} J_{u}^{a}(x) \mathcal{O}_{\lambda}(y)\right\rangle=-\bar{\phi}\left(T^{a}\right)_{21} \delta^{3}(x-y),
\end{gathered}
$$

$-\left\langle\partial_{t} J_{t}^{a}(x) \mathcal{O}_{\phi}(y)\right\rangle+\left\langle\partial_{u} J_{u}^{a}(x) \mathcal{O}_{\phi}(y)\right\rangle=-\bar{\phi}\left(T^{a}\right)_{22} \delta^{3}(x-y)$.

These are of course nothing other than the Ward identities relating the two-point functions of currents associated to broken generators to the VEVs of the operators that break the symmetry. In particular, the relations (3.17)-(3.18) are the usual identities relating the two-point function of the divergence of a conserved current and a scalar operator to the symmetry breaking VEV of the operator. This kind of Ward identity has been already realized holographically; see e.g. $[13,14]$ (and more recently also [17]).

Of more interest is the identity (3.15), which is nontrivial due to the fact that we allow the temporal component of a current ( $J_{i}^{3}$ here) to have a nontrivial VEV. We assume that this Lorentz symmetry breaking VEV is permitted by the presence of a chemical potential, though in our holographic setup this is not technically necessary (indeed $\mu$ does not explicitly appear anywhere in the above expressions). In addition, note that the identity (3.16) is consistently trivial since the spatial (longitudinal) components of the same current cannot get a VEV, because that would violate the invariance under spatial rotations.
The above Ward identities imply the presence of Goldstone bosons, i.e. of massless modes in the spectrum. More precisely, we see that in order to satisfy the identities (3.15)-(3.18), the Fourier transformed correlators $\left\langle J_{t}^{a} J_{t}^{b}\right\rangle(\omega, k), \quad\left\langle J_{t}^{a} \mathcal{O}_{\lambda}\right\rangle(\omega, k)$ and similar ones must be singular when the energy $\omega$ and the momentum $k_{u}$ go to zero. Indeed, in Fourier space (3.15) reads

$$
-i \omega\left\langle J_{t}^{a} J_{t}^{b}\right\rangle+i k_{u}\left\langle J_{u}^{a} J_{t}^{b}\right\rangle=-i \bar{A}^{3} f^{a b 3} .
$$

We thus deduce the presence of massless poles in all of these correlators. This additional Ward identity, which defines [3] type B Goldstone bosons, requires the dispersion relation to be quadratic [16], with the following argument.

In the Lorentz-invariant case, the dispersion relation of a Goldstone boson is trivially determined from a Ward identity similar to (3.17)-(3.18), giving

$$
\left\langle J_{i} \mathcal{O}\right\rangle \propto \frac{k_{i}}{\omega^{2}-k_{u}^{2}} .
$$

In the lack of Lorentz invariance, we have to consider two different situations, one in which time-reversal invariance is preserved and one in which it is broken. When it is preserved, for small values of $\omega$ and $k_{u}$ we can admit

$$
\left\langle J_{t} \mathcal{O}\right\rangle \simeq \frac{\tilde{T} \omega}{\omega^{2}-c_{s}^{2} k^{2}}, \quad\left\langle J_{u} \mathcal{O}\right\rangle \simeq \frac{\tilde{U} k_{u}}{\omega^{2}-c_{s}^{2} k^{2}}
$$

while when it is broken, we can have

$$
\left\langle J_{t} \mathcal{O}\right\rangle \simeq \frac{\bar{T}}{M \omega-k^{2}}, \quad\left\langle J_{u} \mathcal{O}\right\rangle \simeq \frac{\tilde{U} k_{u}}{M \omega-k^{2}},
$$

where $\bar{T}, \tilde{T}, \tilde{U}, c_{s}$ and $M$ are constants. Note now that (3.19) breaks time-reversal invariance for the currents $J_{i}^{1}$ and $J_{i}^{2}$, and actually requires $\bar{T} \neq 0$ as it was proved in [16]. This implies that (3.15) and (3.17) lead to quadratic dispersion relations, $\omega \simeq \frac{k^{2}}{M}$ with $M \equiv \bar{T} / \bar{\phi}$. On the other hand for the Goldstone boson contributing to (3.18), which is of type A [3], the time-reversal invariance is still preserved and the dispersion relation is linear (assuming $\tilde{U}$ has a finite limit for vanishing momentum), but with velocity depending on the ratio $\tilde{U} / \tilde{T} \equiv c_{s}^{2}$.

Without specifying the unknown nonlocal functions $h^{a b}(\partial), f^{a}(\partial)$, etc., we cannot go further and, for instance, find the exact expression for $\bar{T}, \tilde{T}, \tilde{U}$ for all the massless (and light) excitations. In the present model this is of course in principle possible (see [9] for a finite temperature analysis), but would imply solving the equations of motion for the fluctuations in the bulk. This in turn would necessitate finding the backreacted geometry. The point in the present article is to exploit to its limits the technique of holographic renormalization, i.e. to extract the maximal information on the system purely from boundary 
considerations. Perhaps not unexpectedly, we found exactly the same information that can be gathered from Ward identities that apply to the system.

In Appendix B we compute the same correlators in the field theoretical toy model of $[11,12]$, where both the local and the nonlocal parts of the correlators can be made explicit.

\section{CONCLUSIONS AND PERSPECTIVES}

We set out to compute two-point correlators through holographic renormalization, in a strongly coupled field theory model with a global $U(2)$ symmetry, where a chemical potential breaks boost invariance explicitly. We studied the consequences of the spontaneous breaking of $U(2)$ to $U(1)$. Because of the broken Lorentz symmetry, not only scalar operators are allowed to acquire symmetry breaking VEVs, but also temporal components of conserved currents.

We have obtained the general form of the renormalized action up to quadratic order, that is, the generating functional for one- and two-point correlation functions. In this expression, we have kept implicit the nonlocal functions that are established by imposing regularity conditions of the fluctuations in the deep bulk. Nevertheless, we were able to extract information on the light spectrum by showing that some linear combinations of the correlators are completely determined by local terms, and actually realize holographically the Ward identities associated to the broken symmetries.

We have thus established that the system must have Goldstone excitations associated to the three broken generators. However, since we have also shown that $J_{t}^{3}$, and thus $Q^{3}$, has a VEV, we observe that we are exactly in a situation where so-called type B Goldstone bosons [3] arise, i.e. when the commutator of two charges has a nonvanishing VEV. Here, we have that $\left\langle\left[Q^{1}, Q^{2}\right]\right\rangle \neq 0$, so that only one massless excitation is associated to these two broken charges. Furthermore, we have given an argument for this massless excitation to have a quadratic dispersion relation. The remaining broken generator $Q^{0}-Q^{3}$ is not involved in commutators with nontrivial VEVs, and hence gives rise to a type A Goldstone boson, which in this case is expected to have a linear dispersion relation, but with a velocity smaller than $c$ that would be determined by the explicit expressions of the nonlocal parts of the correlators. Moreover, we expect the type B Goldstone boson to be accompanied by an almost Goldstone boson, i.e. a light mode whose mass is related to the coefficient of the quadratic dispersion relation of its partner [4].

We stress once more that in order to obtain quantitative results on all these dispersion relations, we have to compute the nonlocal functions that we have left unspecified in (3.4). The poles in these functions will give us the dispersion relations of the massless modes, together with all the rest of the massive spectrum. In order to do that, we would need first to have a background which is reliable down to the deep bulk. Performing the backreaction, also on the metric, would then be necessary. One should be warned though that in the present zero-temperature setup, this would most inevitably lead to a singular geometry. That should however not prevent us from imposing boundary conditions in the form of boundedness of the fluctuations. Any other stratagem to avoid the singularity would introduce a new scale to the problem, as for instance a finite temperature. That has been done in [9], where however the backreaction is not studied, thus limiting the analysis to situations where the temperature and the chemical potential are roughly at the same scale.

Considering finally generalizations, it is obviously rather straightforward to generalize our discussion to higher dimensions. It would be interesting to investigate, in a fully backreacted model, at zero or nonzero temperature, also correlators involving the stress-energy tensor, and possibly in supersymmetric extensions of such models, whether also the dispersion relations of the Goldstino can be modified, extending the analysis of $[18,19]$.

\section{ACKNOWLEDGMENTS}

We are grateful to Matteo Bertolini, Daniele Musso and Diego Redigolo for useful discussions and feedback on the manuscript, and Manuela Kulaxizi and Andrei Parnachev for interesting comments. This research is supported in part by IISN-Belgium (convention 4.4503.15), by the "Communauté Française de Belgique" through the ARC program and by a "Mandat d'Impulsion Scientifique" of the F. R. S.-Fonds de la Recherche Scientifique (FNRS). R. A. is a Senior Research Associate of the F. N. R. S. (Belgium). The work of A.Me. is supported by the NWO Vidi grant.

\section{APPENDIX A: ALTERNATIVE SPLITTING OF THE VECTOR DEGREES OF FREEDOM}

In this appendix we perform, for completeness, a different splitting of the vector degrees of freedom. Instead of (2.10), we split the vector into transverse and longitudinal parts with respect to all spacetime components:

$$
A_{i}^{a}=a_{i}^{T a}+\partial_{i} a^{L a}, \quad \partial_{i} a_{i}^{T a}=0 .
$$

The regularized action (2.9) then reads, after the substitutions,

$$
\begin{aligned}
S_{\text {reg }}= & -\int_{z=\epsilon} d^{3} x\left\{\bar{A}^{0} a_{t}^{T 0}+\bar{A}^{3} a_{t}^{T 3}\right. \\
& -\frac{2}{z} \bar{\phi}\left(\phi+\phi^{\dagger}\right)-\frac{1}{2} a_{i}^{T a} \partial_{z} a_{i}^{T a} \\
& +\frac{1}{2} \bar{A}^{3} f^{a b 3} a^{L a}\left(a_{t}^{T b}-z \partial_{z} a_{t}^{T b}+\partial_{t} a^{L b}-z \partial_{z} \partial_{t} a^{L b}\right) \\
& +\frac{i}{2 z} \bar{\phi} a^{L a}\left[\left(T^{a}\right)_{21}\left(2 \lambda-z \partial_{z} \lambda\right)-\left(T^{a}\right)_{12}\left(2 \lambda^{\dagger}-z \partial_{z} \lambda^{\dagger}\right)\right. \\
& \left.+\left(T^{a}\right)_{22}\left(2 \phi-2 \phi^{\dagger}-z \partial_{z} \phi+z \partial_{z} \phi^{\dagger}\right)\right] \\
& \left.-\frac{1}{2 z^{2}}\left(\lambda^{\dagger} \partial_{z} \lambda+\lambda \partial_{z} \lambda^{\dagger}+\phi^{\dagger} \partial_{z} \phi+\phi \partial_{z} \phi^{\dagger}\right)\right\} . \quad \text { (A2) }
\end{aligned}
$$


The near-boundary expansions of the vector fields are now

$$
\begin{aligned}
& a_{i}^{T a}=a_{i(0)}^{T a}+a_{i(1)}^{T a} z+\cdots \\
& a^{L a}=a_{(0)}^{L a}+a_{(1)}^{L a} z+\cdots
\end{aligned}
$$

and the renormalized action reads

$$
\begin{aligned}
S_{\text {ren }}= & -\int d^{3} x\left\{\bar{A}^{0} a_{t(0)}^{T 0}+\bar{A}^{3} a_{t(0)}^{T 3}-2 \bar{\phi}\left(\phi_{(0)}+\phi_{(0)}^{\dagger}\right)\right. \\
& -\frac{1}{2} a_{i(0)}^{T a} a_{i(1)}^{T a}+\frac{1}{2} \bar{A}^{3} f^{a b 3} a_{(0)}^{L a}\left(a_{t(0)}^{T b}+\partial_{t} a_{(0)}^{L b}\right) \\
& +\frac{i}{2} \bar{\phi} a_{(0)}^{L a}\left[\left(T^{a}\right)_{21} \lambda_{(0)}-\left(T^{a}\right)_{12} \lambda_{(0)}^{\dagger}\right. \\
& \left.+\left(T^{a}\right)_{22}\left(\phi_{(0)}-\phi_{(0)}^{\dagger}\right)\right] \\
& \left.-\frac{1}{2}\left(\lambda_{(0)}^{\dagger} \lambda_{(1)}+\lambda_{(0)} \lambda_{(1)}^{\dagger}+\phi_{(0)}^{\dagger} \phi_{(1)}+\phi_{(0)} \phi_{(1)}^{\dagger}\right)\right\} .
\end{aligned}
$$

The residual gauge transformations act on the transverse and longitudinal modes as

$$
\begin{aligned}
\delta a_{i(0)}^{T a} & =0, \quad \delta a_{i(1)}^{T a}=f^{a b 3} \bar{A}^{3}\left(\eta_{t i}-\frac{\partial_{t} \partial_{i}}{\partial_{j}^{2}}\right) \alpha^{b}, \\
\delta a_{(0)}^{L a} & =\alpha^{a}, \quad \delta a_{(1)}^{L a}=f^{a b 3} \bar{A}^{3} \frac{\partial_{t}}{\partial_{i}^{2}} \alpha^{b} .
\end{aligned}
$$

Note that the gauge transformations of the VEV fluctuations are nonlocal with this choice of splitting. The gauge-invariant combinations are

$$
\begin{aligned}
& \lambda_{(1)}-i \bar{\phi}\left(T^{a}\right)_{12} a_{(0)}^{L a}, \\
& \phi_{(1)}-i \bar{\phi}\left(T^{a}\right)_{22} a_{(0)}^{L a}, \\
& a_{i(1)}^{T a}-\bar{A}^{3} f^{a b 3}\left(\eta_{t i}-\frac{\partial_{t} \partial_{i}}{\partial_{j}^{2}}\right) a_{(0)}^{L b} .
\end{aligned}
$$

The expressions of the VEVs in terms of the sources are then

$$
\begin{aligned}
\lambda_{(1)}= & i \bar{\phi}\left(T^{a}\right)_{12} a_{(0)}^{L a}+f(\partial) \lambda_{(0)}+\tilde{f}^{a}(\partial) a_{t(0)}^{T a}, \\
\phi_{(1)}= & i \bar{\phi}\left(T^{a}\right)_{22} a_{(0)}^{L a}+g(\partial) \phi_{(0)}+\tilde{g}^{a}(\partial) a_{t(0)}^{T a}, \\
a_{t(1)}^{T a}= & -\bar{A}^{3} f^{a b 3}\left(1+\frac{\partial_{t}^{2}}{\partial_{i}^{2}}\right) a_{(0)}^{L b}+h^{a b}(\partial) a_{t(0)}^{T b} \\
& +k^{a}(\partial) \lambda_{(0)}+l^{a}(\partial) \phi_{(0)} \\
& +k^{a}(\partial)^{*} \lambda_{(0)}^{\dagger}+l^{a}(\partial)^{*} \phi_{(0)}^{\dagger}, \\
a_{u(1)}^{T a}= & -\bar{A}^{3} f^{a b 3} \frac{\partial_{t} \partial_{u}}{\partial_{i}^{2}} a_{(0)}^{L b}+m^{a b}(\partial) a_{u(0)}^{T b},
\end{aligned}
$$

where we have used the same letters for the unknown nonlocal functions, which however will be possibly different from the ones in (3.4).

Eliminating all the VEVs, $S_{\text {ren }}$ then reads

$$
\begin{aligned}
S_{\text {ren }}= & -\int d^{3} x\left\{\bar{A}^{0} a_{t(0)}^{T 0}+\bar{A}^{3} a_{t(0)}^{T 3}-2 \bar{\phi}\left(\phi_{(0)}+\phi_{(0)}^{\dagger}\right)-\frac{1}{2} a_{u(0)}^{T a} m^{a b}(\partial) a_{u(0)}^{T b}\right. \\
& +\frac{1}{2} a_{t(0)}^{T a} h^{a b}(\partial) a_{t(0)}^{T b}-\frac{1}{2} \bar{A}^{3} f^{a b 3}\left(2 a_{t(0)}^{T a}+\partial_{t} a_{(0)}^{L a}\right) a_{(0)}^{L b} \\
& +i \bar{\phi} a_{(0)}^{L a}\left[\left(T^{a}\right)_{21} \lambda_{(0)}-\left(T^{a}\right)_{12^{2}} \lambda_{(0)}^{\dagger}+\left(T^{a}\right)_{22}\left(\phi_{(0)}-\phi_{(0)}^{\dagger}\right)\right] \\
& -\frac{1}{2} \lambda_{(0)}^{\dagger}\left(f(\partial)+f(\partial)^{*}\right) \lambda_{(0)}-\frac{1}{2} \phi_{(0)}^{\dagger}\left(g(\partial)+g(\partial)^{*}\right) \phi_{(0)} \\
& -\frac{1}{2}\left[\lambda_{(0)}^{\dagger}\left(\tilde{f}^{a}(\partial)+k^{a}(\partial)^{*}\right)+\lambda_{(0)}\left(\tilde{f}^{a}(\partial)^{*}+k^{a}(\partial)\right)\right] a_{t(0)}^{T a} \\
& -\frac{1}{2}\left[\phi_{(0)}^{\dagger}\left(\left(\tilde{g}^{a}(\partial)+l^{a}(\partial)^{*}\right)+\phi_{(0)}\left(\tilde{g}^{a}(\partial)^{*}+l^{a}(\partial)\right)\right] a_{t(0)}^{T a}\right\} .
\end{aligned}
$$

The coupling of the sources to the currents is now

$$
\int_{\partial \mathrm{AdS}} d^{3} x\left(a_{t(0)}^{T a} J_{t}^{T a}-a_{u(0)}^{T a} J_{u}^{T a}+a_{(0)}^{L a} \partial_{i} J_{i}^{a}\right) .
$$

The Ward identities are then directly derived as 


$$
\begin{aligned}
\left\langle\partial_{i} J_{i}^{a}(x) J_{t}^{T b}(y)\right\rangle & =-i \frac{\delta^{2} S_{\text {ren }}}{\delta a_{(0)}^{L a}(x) \delta a_{t(0)}^{T b}(y)} \\
& =-i \bar{A}^{3} f^{a b 3} \delta^{3}(x-y), \\
\left\langle\partial_{i} J_{i}^{a}(x) \mathcal{O}_{\lambda}(y)\right\rangle & =-i \frac{\delta^{2} S_{\text {ren }}}{\delta a_{(0)}^{L a}(x) \delta \lambda_{(0)}(y)} \\
& =-\bar{\phi}\left(T^{a}\right)_{21} \delta^{3}(x-y), \\
\left\langle\partial_{i} J_{i}^{a}(x) \mathcal{O}_{\phi}(y)\right\rangle & =-i \frac{\delta^{2} S_{\text {ren }}}{\delta a_{(0)}^{L a}(x) \delta \phi_{(0)}(y)} \\
& =-\bar{\phi}\left(T^{a}\right)_{22} \delta^{3}(x-y),
\end{aligned}
$$

reproducing exactly the results of (3.15)-(3.18). The other correlators are purely in terms of the nonlocal parts, for instance

$$
\begin{aligned}
\left\langle J_{t}^{T a}(x) \mathcal{O}_{\lambda}(y)\right\rangle & =-i \frac{\delta^{2} S_{\text {ren }}}{\delta a_{t(0)}^{T a}(x) \delta \lambda_{(0)}(y)} \\
& =-\frac{i}{2}\left(\tilde{f}^{a}(\partial)^{*}+k^{a}(\partial)\right) \delta^{3}(x-y)
\end{aligned}
$$

and similarly for the others. In the main text we have opted for the splitting (2.10) because it more directly reflects the loss of boost invariance, and it avoids the nonlocal expression in (A6).

\section{APPENDIX B: CORRELATORS AND WARD IDENTITIES IN A FIELD THEORY MODEL}

In this appendix we discuss the field theory model of $[11,12]$ which shares the same symmetries and pattern of symmetry breaking as the holographic model discussed in this article. In the weak coupling limit, we can compute the two-point correlators at tree level, verify once more the Ward identities but also extract the dispersion relations and the spectrum.

The model consists of just a complex scalar doublet $\Phi$ of a $U(2)$ global symmetry, with a chemical potential $\mu$ for the Abelian $U(1)$ current. The action is

$$
\begin{aligned}
S= & \int d^{3} x\left[\left(\partial_{t}+i \mu\right) \Phi^{\dagger}\left(\partial_{t}-i \mu\right) \Phi-\partial_{u} \Phi^{\dagger} \partial_{u} \Phi\right. \\
& \left.-M^{2} \Phi^{\dagger} \Phi-\lambda\left(\Phi^{\dagger} \Phi\right)^{2}\right] .
\end{aligned}
$$

The conserved currents are given by

$$
J_{i}^{a}=i \Phi^{\dagger} T^{a} \partial_{i} \Phi-i \partial_{i} \Phi^{\dagger} T^{a} \Phi-2 \mu \delta_{t i} \Phi^{\dagger} T^{a} \Phi .
$$

One can verify that they satisfy the $U(2)$ current algebra, for any value of $\mu$ (which is real).
When $\mu^{2}>M^{2}$ (i.e. always if $M^{2}<0$ ) the theory settles in a vacuum where the global symmetry is broken down to a $U(1)$; for definiteness we choose

$$
\Phi_{v}=\left(\begin{array}{l}
0 \\
v
\end{array}\right), \quad v^{2}=\frac{\mu^{2}-M^{2}}{2 \lambda} .
$$

We can now consider the small fluctuations around the vacuum:

$$
\Phi=\Phi_{v}+\left(\begin{array}{c}
\xi \\
\varphi
\end{array}\right) .
$$

Expanding the action to quadratic order we obtain the following tree-level propagators:

$$
\begin{aligned}
\left\langle\xi(\omega, k) \xi^{\dagger}(-\omega,-k)\right\rangle & =\frac{i\left(\omega^{2}-k^{2}-2 \mu \omega\right)}{\left(\omega^{2}-\omega_{\xi+}^{2}\right)\left(\omega^{2}-\omega_{\xi_{-}}^{2}\right)}, \\
\langle\xi(\omega, k) \xi(-\omega,-k)\rangle & =\left\langle\xi^{\dagger}(\omega, k) \xi^{\dagger}(-\omega,-k)\right\rangle=0, \\
\left\langle\varphi(\omega, k) \varphi^{\dagger}(-\omega,-k)\right\rangle & =\frac{i\left(\omega^{2}-k^{2}-2 \mu \omega-2 \lambda v^{2}\right)}{\left(\omega^{2}-\omega_{\varphi+}^{2}\right)\left(\omega^{2}-\omega_{\varphi-}^{2}\right)}, \\
\langle\varphi(\omega, k) \varphi(-\omega,-k)\rangle & =\left\langle\varphi^{\dagger}(\omega, k) \varphi^{\dagger}(-\omega,-k)\right\rangle \\
& =\frac{2 i \lambda v^{2}}{\left(\omega^{2}-\omega_{\varphi+}^{2}\right)\left(\omega^{2}-\omega_{\varphi-}^{2}\right)},
\end{aligned}
$$

with the dispersion relations

$$
\begin{aligned}
& \omega_{\xi \pm}^{2}=k^{2}+2 \mu^{2} \pm 2 \mu \sqrt{\mu^{2}+k^{2}}, \\
& \omega_{\varphi \pm}^{2}=k^{2}+3 \mu^{2}-M^{2} \pm \sqrt{\left(3 \mu^{2}-M^{2}\right)^{2}+4 \mu^{2} k^{2}},
\end{aligned}
$$

which at small momenta $k$ give

$$
\begin{aligned}
\omega_{\xi+} & =2 \mu+\cdots, \\
\omega_{\xi-} & =\frac{k^{2}}{2 \mu}+\cdots, \\
\omega_{\varphi+} & =\sqrt{6 \mu^{2}-2 M^{2}}+\cdots, \\
\omega_{\varphi-} & =\sqrt{\frac{\mu^{2}-M^{2}}{3 \mu^{2}-M^{2}}} k+\cdots
\end{aligned}
$$

We see that we have all the variety of massless and massive modes: $\omega_{\varphi-}$ for a type A Goldstone boson with $c_{s}<1, \omega_{\xi-}$ for a type B Goldstone boson, $\omega_{\xi+}$ for its massive partner and $\omega_{\varphi+}$ for a Higgs-like massive excitation. Furthermore, we can compute the VEV of the currents and find, e.g., $\left\langle J_{t}^{3}\right\rangle=\mu v^{2}$, which correctly vanishes when $\mu \rightarrow 0$.

In the broken symmetry vacuum, the currents can also be expanded at the linear order: 


$$
\begin{aligned}
J_{i}^{0} & =\frac{i}{2} v \partial_{i}\left(\varphi-\varphi^{\dagger}\right)-\delta_{t i} \mu v\left(\varphi+\varphi^{\dagger}\right), \\
J_{i}^{1} & =\frac{i}{2} v \partial_{i}\left(\xi-\xi^{\dagger}\right)-\delta_{t i} \mu v\left(\xi+\xi^{\dagger}\right), \\
J_{i}^{2} & =-\frac{1}{2} v \partial_{i}\left(\xi+\xi^{\dagger}\right)-i \delta_{t i} \mu v\left(\xi-\xi^{\dagger}\right), \\
J_{i}^{3} & =-J_{i}^{0} .
\end{aligned}
$$

It is then straightforward to compute the correlators analogous to the ones in (3.8)-(3.14).

Let us for instance compute one such set of correlators:

$\left\langle J_{t}^{1}(\omega, k) \xi(-\omega,-k)\right\rangle=\frac{1}{2} v(\omega-2 \mu) \frac{i\left(\omega^{2}-k^{2}+2 \mu \omega\right)}{\left(\omega^{2}-\omega_{\xi+}^{2}\right)\left(\omega^{2}-\omega_{\xi-}^{2}\right)}$,

$\left\langle J_{u}^{1}(\omega, k) \xi(-\omega,-k)\right\rangle=\frac{1}{2} v k_{u} \frac{i\left(\omega^{2}-k^{2}+2 \mu \omega\right)}{\left(\omega^{2}-\omega_{\xi+}^{2}\right)\left(\omega^{2}-\omega_{\xi-}^{2}\right)}$, which are clearly nonlocal since they contain the poles for the type B Goldstone boson and its massive partner. However the combination

$$
\begin{aligned}
& -i \omega\left\langle J_{t}^{1}(\omega, k) \xi(-\omega,-k)\right\rangle \\
& \quad+i k_{u}\left\langle J_{u}^{1}(\omega, k) \xi(-\omega,-k)\right\rangle=\frac{v}{2}
\end{aligned}
$$

is constant, by virtue of a Ward identity similar to the one in (3.17), for the above correlator.

All the other Ward identities can be similarly checked. In this weakly coupled model however, as we have seen, we have access also to the individual correlators and hence to the nonlocal parts that contain complete information on the spectrum and on the dispersion relations.
[1] H. B. Nielsen and S. Chadha, On how to count Goldstone bosons, Nucl. Phys. B105, 445 (1976).

[2] H. Watanabe and T. Brauner, On the number of NambuGoldstone bosons and its relation to charge densities, Phys. Rev. D 84, 125013 (2011).

[3] H. Watanabe and H. Murayama, Unified Description of Nambu-Goldstone Bosons without Lorentz Invariance, Phys. Rev. Lett. 108, 251602 (2012).

[4] A. Kapustin, Remarks on nonrelativistic Goldstone bosons, arXiv:1207.0457.

[5] H. Watanabe and H. Murayama, Effective Lagrangian for Nonrelativistic Systems, Phys. Rev. X 4, 031057 (2014).

[6] J. M. Maldacena, The large N limit of superconformal field theories and supergravity, Adv. Theor. Math. Phys. 2, 231 (1998); J. M. Maldacena, Int. J. Theor. Phys. 38, 1113 (1999).

[7] E. Witten, Anti-de Sitter space and holography, Adv. Theor. Math. Phys. 2, 253 (1998).

[8] S. S. Gubser, I. R. Klebanov, and A. M. Polyakov, Gauge theory correlators from noncritical string theory, Phys. Lett. B 428, 105 (1998).

[9] I. Amado, D. Arean, A. Jimenez-Alba, K. Landsteiner, L. Melgar, and I. S. Landea, Holographic type II Goldstone bosons, J. High Energy Phys. 07 (2013) 108.

[10] V. G. Filev, C. V. Johnson, and J. P. Shock, Universal holographic chiral dynamics in an external magnetic field, J. High Energy Phys. 08 (2009) 013.
[11] V. A. Miransky and I. A. Shovkovy, Spontaneous Symmetry Breaking with Abnormal Number of Nambu-Goldstone Bosons and Kaon Condensate, Phys. Rev. Lett. 88, 111601 (2002).

[12] T. Schäfer, D. T. Son, M. A. Stephanov, D. Toublan, and J. J. M. Verbaarschot, Kaon condensation and Goldstone's theorem, Phys. Lett. B 522, 67 (2001).

[13] M. Bianchi, D.Z. Freedman, and K. Skenderis, How to go with an RG flow, J. High Energy Phys. 08 (2001) 041.

[14] M. Bianchi, D. Z. Freedman, and K. Skenderis, Holographic renormalization, Nucl. Phys. B631, 159 (2002).

[15] J. Goldstone, A. Salam, and S. Weinberg, Broken symmetries, Phys. Rev. 127, 965 (1962).

[16] T. Brauner, Goldstone bosons in the presence of charge density, Phys. Rev. D 75, 105014 (2007).

[17] R. Argurio, D. Musso, and D. Redigolo, Anatomy of new SUSY breaking holographic RG flows, J. High Energy Phys. 03 (2015) 086.

[18] R. Argurio, M. Bertolini, L. Di Pietro, F. Porri, and D. Redigolo, Supercurrent multiplet correlators at weak and strong coupling, J. High Energy Phys. 04 (2014) 123.

[19] R. Argurio, M. Bertolini, D. Musso, F. Porri, and D. Redigolo, The holographic Goldstino, Phys. Rev. D 91, 126016 (2015). 